\title{
EBL measurements through the TeV gamma-ray spectra of Low- and High-red shifted AGN
}

\section{V.Y. Sinitsyna*}

P.N. Lebedev Physical Institute, Leninsky pr. 53, Moscow, Russia

E-mail: sinits@sci.lebedev.ru

\section{V.G. Sinitsyna}

P.N. Lebedev Physical Institute, Leninsky pr. 53, Moscow, Russia

E-mail: sinits@sci.lebedev.ru

\begin{abstract}
The detection of $\mathrm{TeV}$ gamma-ray sources at high red-shifts namely NGC1275 ( $\mathrm{z}=0.0179)$, Mkn421 ( $\mathrm{z}=0.031), \operatorname{Mkn} 501 \quad(\mathrm{z}=0.034), M k n 180 \quad(\mathrm{z}=0.046), 3 \mathrm{c} 382 \quad(\mathrm{z}=0.0578), 4 \mathrm{c}+31.63$ $(\mathrm{z}=0.295)$, OJ $287(\mathrm{z}=0.306), 3 \mathrm{c} 454.3(\mathrm{z}=0.859), 4 \mathrm{c}+55.17(\mathrm{z}=0.896)$ and $1739+522(\mathrm{z}=1.375)$ is the evidence of less average spectral density of Extragalactic Background Light and thus the less star formation rate at early evolution stage, than it is previously believed. Also, the possible explanation of the detected very high energy gamma-emission from the distant AGNi is the re-scattering of primary TeV-photons on the Dark Matter particles, so called WISP - weakly interacting slim particles. The axion-like particles has been considered to be a candidate for such weakly interacting slim particles.
\end{abstract}

Frontiers of Fundamental Physics 14 - FFP14,

15-18 July 2014

Aix Marseille University (AMU) Saint-Charles Campus, Marseille

${ }^{*}$ Speaker. 


\section{Introduction}

The cosmological processes, connecting the physics of matter in Active Galactic Nuclei will be observed in the energy spectrum of electromagnetic radiation. The detection of the very high energy $\gamma$-ray sources at the redshifts $\mathrm{z}=0.0179$ to $\mathrm{z}=1.375$ with SHALON telescope gives an opportunity to constrain extragalactic background light (EBL) density, based on modification to $\gamma$-ray spectra and thus it will help to reconstruct the the cosmological history of the EBL.

\section{SHALON telescope}

The SHALON mirror Cherenkov telescope system (Fig. 1) is designed to detect $\gamma$-rays from local sources in the energy range from $800 \mathrm{GeV}$ to $100 \mathrm{TeV}$. The SHALON $\gamma$-ray telescopes are located at an altitude of $3340 \mathrm{~m}$ above sea level each of which has a composite mirror with an area of $11.2 \mathrm{~m}^{2}$. The detector array consisting of 144 FEU-85 photomultipliers assembled into a square array and mounted at the mirror focus has characteristics sufficient to record information about the shower structure in the energy range under consideration. The detector has the largest field of view in the world, $>8^{\circ}$. This allows one to monitor the background from charged cosmic-ray particles and the atmospheric transparency continuously during observations and expands the area of observation and, hence, the efficiency of observations [1]. The technique for simultaneously obtaining information about the cosmic-ray background and the showers initiated by $\gamma$-rays is unique and has been applied in the SHALON experiment from the very beginning of its operation [1]. This technique serves to increase the useful source tracking time and, what is particularly important, such source and background observation conditions as the thickness and state of the atmosphere remain the same. In addition, the wide field of view allows recording the off-center showers arriving at distances of more than $30 \mathrm{~m}$ from the telescope axis completely and almost without any distortions; they account for more $90 \%$ of all the showers recorded by the telescope. During a primary analysis, the primary particle arrival direction is determined with an accuracy up to $\leq 0.1^{\circ}$. The subsequent analysis specially developed for the SHALON telescopes and based on Tikhonov regularization method [2,3] improves the accuracy to a value of less than $0.01^{\circ}[4]$.

During the period 1992 - 2014 SHALON has been used for observations of extragalactic sources of different type. The main parameters obtained for each of sources are presented at table 1 $[5,6,7,8]$. Observations of distant metagalactic sources have shown that the Universe is more transparent to very high-energy $\gamma$-rays than previously believed.
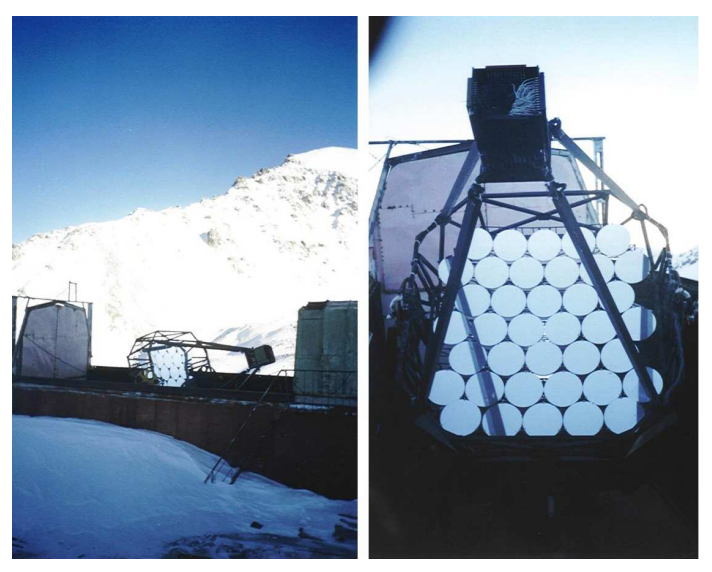

Figure 1: SHALON-1 mirror Cherenkov telescope in the observatory. 
Table 1: The catalogue of and extragalactic $\gamma$-ray sources by SHALON with parameters for spectrum fitting in form of power low with exponential cutoff $F(>E) \propto E^{k_{\gamma}} \times \exp \left(-E / E_{\text {cutoff }}\right)$.

\begin{tabular}{cccccc}
\hline Sources & Observable flux $^{a}$ & $k_{\gamma}$ & $E_{\text {cutoff }}, \mathrm{TeV}$ & Distance, z & Type \\
\hline NGC 1275 & $(0.78 \pm 0.05)$ & $-2.18 \pm 0.12$ & $32 \pm 7$ & 0.018 & Seyfert \\
SN2006 gy & $(3.71 \pm 0.65)$ & $-3.10 \pm 0.30$ & $4.4 \pm 1.9$ & 0.019 & SN \\
Mkn 421 & $(0.63 \pm 0.05)$ & $-1.51 \pm 0.18$ & $10 \pm 3$ & 0.031 & BL Lac \\
Mkn 501 & $(0.86 \pm 0.06)$ & $-1.48 \pm 0.15$ & $11 \pm 3$ & 0.034 & BL Lac \\
Mkn 180 & $(0.65 \pm 0.09)$ & $-1.84 \pm 0.15$ & $7.3 \pm 2.2$ & 0.046 & BL Lac \\
3c382 & $(0.91 \pm 0.14)$ & $-1.08 \pm 0.11$ & $21 \pm 7.0$ & 0.0578 & BLRG \\
4c+31.63 & $(0.73 \pm 0.16)$ & $-1.13 \pm 0.16$ & $10.4 \pm 3.2$ & 0.295 & FSRQ \\
OJ 287 & $(0.26 \pm 0.07)$ & $-1.14 \pm 0.11$ & $9.5 \pm 1.2$ & 0.306 & BL Lac \\
3c454.3 & $(0.43 \pm 0.07)$ & $-0.52 \pm 0.12$ & $6.2 \pm 1.0$ & 0.859 & FSRQ \\
4c+55.17 & $(0.90 \pm 0.16)$ & $-1.40 \pm 0.15$ & $5.4 \pm 2.1$ & 0.896 & FSRQ \\
1739+522 & $(0.49 \pm 0.05)$ & $-0.50 \pm 0.18$ & $6.1 \pm 1.2$ & 1.375 & FSRQ \\
B2 0242+43 & $(0.58 \pm 0.20)$ & - & - & 2.243 & FSRQ \\
B2 0743+25 & $(0.37 \pm 0.16)$ & - & - & 2.979 & FSRQ \\
\hline \multicolumn{7}{c}{ Integral flux at energy $>800 \mathrm{GeV}$ in units of $10^{-12} \mathrm{~cm}^{-2} \mathrm{~s}^{-1}$} &
\end{tabular}

\section{Extragalactic Background Light}

$\mathrm{TeV} \gamma$-rays, radiated by distant sources, interact with photons of background via $\gamma+\gamma \rightarrow e^{+} e^{-}$ resonant process $[9,10]$, then relativistic electrons can radiate $\gamma$-ray with energies less than of primary $\gamma$-quantum. As a result, primary spectrum of $\gamma$-source is changed, depending on spectrum of background light. So, hard spectra of AGNi with high red shifts of $0.03-1.8$ allow the determination an absorption by EBL and thus its spectrum.

The redshifts of SHALON very high energy $\gamma$-ray sources range from $z=0.018$ to $z=1.375$. Among them bright enough AGNi of BLLac type, Seyfert Galaxy, BLRG and FSRQs (see table 1) those spectra are resolved in the $\mathrm{TeV}$ energy band from $800 \mathrm{GeV}$ up to $\sim 20-50 \mathrm{TeV}$. The fit of a power law with an exponential cutoff function to the measured spectra presented in Table 1.

It has mentioned that the observed spectra are modified by $\gamma$-ray attenuation, i.e. $F_{\text {observed }}(E)=$ $F_{\text {intrinsic }}(E) \times \exp (-\tau(E, z))$ where $\tau(E, z)$ is optical depth for pair creation for a source at redshift $z$, and at an observed energy $E$. According to the definition of the optical opacity the medium influences on the primary source spectrum at $\tau \geq 1$, but for $\tau<1$ the medium is transparent, so the measuring of source spectrum in the both range of $\tau$ can give the intrinsic spectrum of the source to constrain the EBL density. The optical depth for sources at redshifts from 0.018 to 1.375 was calculated with assumption of EBL shapes shown in Fig. 2. We used the EBL shape from Best-fit model and Low-SFR model [12] (lines 1) (Fig. 2) to calculate the attenuated spectrum of Mkn 421, Mkn 501, Mkn 80, 3c382 in assumption of simple power low intrinsic spectrum of the source with spectrum index of $\gamma=1.5 \pm 0.3$, taken from the range of $\tau<1$ (table 1). As NGC 1275 is a nearest source its primary spectrum is weakly influenced by EBL and no significant dependance of spectrum change on models. The shapes of EBL density constrained from the spectra of the high redshift sources $4 c+31.63(z=0.295), 0 J 287(z=0.306), 3 c 454.3(z=0.859), 4 c+55.17(z=0.896)$ and $1739+522(\mathrm{z}=1.375)$ are shown in Fig. 2 with curves $\mathbf{2}, \mathbf{3}$ and $\mathbf{4}$. For these sources the slope of intrinsic integral spectrum is taken $\gamma=1.2 \pm 0.2$. The attenuated spectra for low- and highredshifted sources are presented with Fig. 3 (lines) together with observational data. 

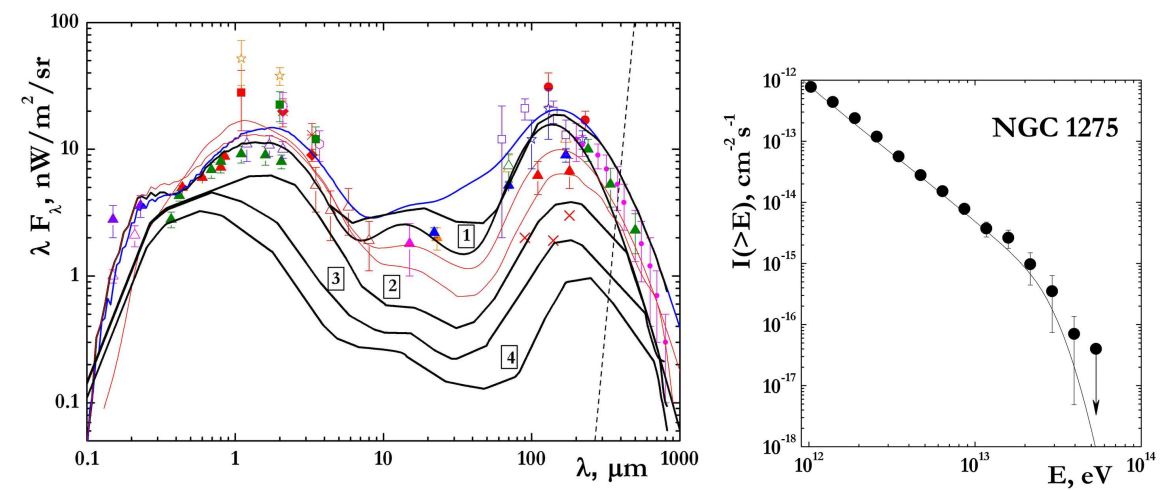

Figure 2: left: Spectral energy distribution of EBL: measurements [11],models [12, 13,14] and EBL shape constrained from observations of the extragalactic sources by SHALON: 1 - NGC 1275 ( $\mathrm{z}=0.018)$, Mkn $421(\mathrm{z}=0.031)$, Mkn 501 ( $\mathrm{z}=0.034)$, Mkn $180(\mathrm{z}=0.046), 3 \mathrm{c} 382(\mathrm{z}=0.0578) ; 2-4 \mathrm{c}+31.63(\mathrm{z}=0.295)$, OJ287 $(\mathrm{z}=0.306) ; 3-3 \mathrm{c} 454.3(\mathrm{z}=0.859), 4 \mathrm{c} 55.17(\mathrm{z}=0.896) ; 4-1739+522(\mathrm{z}=1.375)$. right: Integral energy spectrum of NGC 1275 by SHALON (black points) with spectrum attenuated by EBL (line)
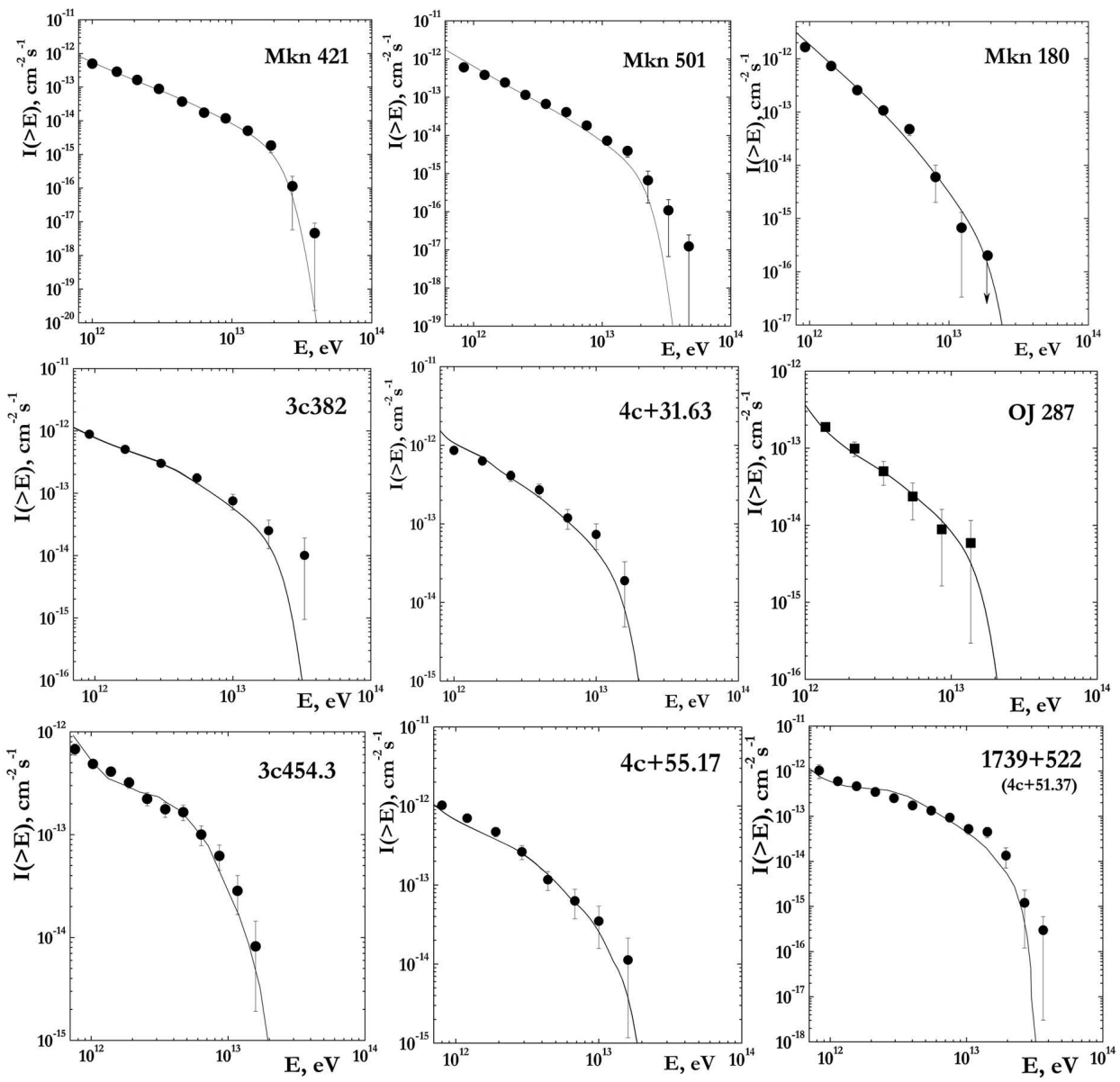

Figure 3: The integral energy spectra for Mkn 421, Mkn 501, Mkn 180, 3c382, 4c+31.63, OJ287, 3c454.4, $4 c+55.17$ and $1739+522$ by SHALON (black points) with spectra attenuated by EBL (lines)

\section{B2 $0242+43$ and B2 $0743+25(z>2)$}

As it discussed in $[9,10]$ and later, at energies of 10 th $\mathrm{GeV}-\mathrm{TeV}$ the $\gamma$-ray spectra of highredshift sources begin to be attenuated by the still poorly known EBL photon field. So, to estimate the cosmic gamma-ray horizon two extremely high-redshifted $(z>2)$ sources from first and second Fermi LAT AGN catalogue are observed by SHALON in autumn-winter period of 2014 year. 
B2 $0242+43$, at redshift $\mathrm{z}=2.243$, is the radio-loud active galaxy classified as a FSRQ. B2 $0242+43$ was observed by SHALON in the period of 2014 for a total of 14 hours. Excess corresponding to a $4.8 \sigma$ [15] was determined with integral flux of $(0.58 \pm 0.20) \times 10^{-12} \mathrm{~cm}^{-2} \mathrm{~s}^{-1}$ also the unexpected high-level flux increase up to the twice over the detected in average was observed at end of November. The surroundings of B2 $0242+43$ of 20' size in radius doesn't contain any candidate for the TeV-emission as well as the GRBs, supernova explosions or other powerful over the wide wavelength range variable sources that could be the sources of the flares detected in the observation period. The list of nearby sources includes two weak radio-objects and one X-ray source didn't confirmed after their detection $[16,17,18]$.

B2 $0743+25$, at redshift $z=2.979$, is the FSRQ from Fermi LAT catalogues. B2 $0743+25$ was observed by SHALON in the period of 2014 for a total of 10 hours. The integral $\gamma$-ray flux of $(0.37 \pm 0.16) \times 10^{-12} \mathrm{~cm}^{-2} \mathrm{~s}^{-1}$ was determined with the significance of $3.9 \sigma$ [15]. The analysis of the region of 20' in radius around B2 $0743+25$ was performed in order to find close powerful sources that could be resolved as this distant quasar $[16,18]$. Among the nearby sources no candidate for the TeV-emission were found as four of quasar objects known only due to identification by V B U magnitude and were not recognized in radio, X-ray or MeV-GeV energies [16].

Further observations and investigations of B2 0242+43, B2 0743+25 and area around these objects are undertaken to establish the reliable source of $\mathrm{TeV} \gamma$-ray emission.

\section{References}

[1] V. G. Sinitsyna, Nuovo Cim. 19C, 965 (1996).

[2] A. N. Tikhonov, V. Ya. Arsenin, Solutions of Ill-Posed Problems (Nauka, Moscow, 1979).

[3] A. V. Goncharskii, A. M. Cherepashchuk, A. G. Yagola, Ill-Posed Problems in Astrophysics (Nauka, Fizmatlit, Moscow, 1985) [in Russian].

[4] V. G. Sinitsyna and V. Yu. Sinitsyna, Astron. Lett. 40(2-3), 75 (2014); 37(9), 621 (2011).

[5] V. G. Sinitsyna et al., Int. J. Mod. Phys. A 29, 7023, 7026, 7029 (2005).

[6] V. G. Sinitsyna et al., Nucl. Phys. B (Proc. Suppl.) 196, 251, 442 (2009); ibid. 175-176, 463 (2008); ibid. 151, 108 (2006); ibid. 122, 247, 409 (2003); ibid. 97, 215 and 219 (2001); ibid. 75A, 352 (1999).

[7] V. G. Sinitsyna, AIP (Conf. Proc.) 515, 205 and 293 (2000).

[8] V.G. Sinitsyna, et al., 2013 J. Phys.: Conf. Ser. 409012112.

[9] A. I. Nikishov, Sov. Phys. JETP, 14, 393 (1962).

[10] R.P. Gould, G.P. Schréder, Phys. Rev. 1551408 (1967).

[11] R. C. Gilmore et al., ArXiv:1104.0671

[12] T. M. Kneiske et al., Astron.\&Astrophys. 386, 1 (2002); 413, 807 (2004).

[13] F. W. Stecker, M. A. Malkan, S. T. Scully, Astrophys. J. 648, 774 (2006).

[14] M. R. Orr, F. Krennrich, E. Dwek, Astrophys. Space Sci. 733, 77 (2011).

[15] T.-P. Li, Y.-Q. Ma, Astrophys. J. 272317 (1983).

[16] M.-P. Veron-Cetty, P. Veron Astron. Astrophys. 518, A10-10 (2010).

[17] I. I. K. Pauliny-Toth et al., Astron. J. 77, 265 (1972).

[18] W . Voges et al., Astron. Astrophys. 349, 389 (1999). 